\title{
Per High Powered Field
}

National Cancer Institute

\section{Source}

National Cancer Institute. Per High Powered Field. NCI Thesaurus. Code C96619.

A unit of measure equal to the instances of an entity per visual field of a microscope set

to its maximum magnification power. 